\title{
Fehler vermeiden und Daten vergleichen
}

Prof. Dr. med. Hans UIrich Rothen, Dr. med. Roger Lussmann, Chantal Britschgi, Ursula Betschart

Mitglieder der Schweizerischen Gesellschaft für Intensivmedizin (SGI)

Vom 14. bis 15. September traf sich die Schweizerische Gesellschaft für Intensivmedizin (SGI) in St. Gallen zu ihrer Jahrestagung 2017. Auch der diesjährigen Ausgabe ging ein Pre-Congress-Day voraus, an dem mit dem ICU-Management-Seminar eine interdisziplinäre und interprofessionelle Veranstaltung stattfand, die sich mit ihrer dritten Ausgabe einen festen Platz im Kalender der Fachgesellschaft gesichert hat.

Von Behandlungsfehlern und deren rechtlichen Folgen über die Komplexität von Intensivstationen bis hin zum immer wichtiger werdenden Benchmarking wurde eine Vielfalt an Themen behandelt, die etwas gemeinsam haben: Sie alle sind wichtig für die Qualitätsförderung der Intensivmedizin in der Schweiz.

\section{Fehler und ihre rechtlichen Konsequenzen}

Die Qualitätsstandards können noch so hoch sein, Standard Operating Procedures und Best-Practice-Leitfäden noch so lückenlos und verständlich, Behandlungsfehler passieren dennoch. Nach den gravierendsten Fehlern stirbt vielleicht sogar ein Patient oder eine Patientin und der Fall wird von den Behörden untersucht. Doch wann muss ein Tod überhaupt gemeldet werden? Für Ärztinnen, Ärzte und Pflegefachpersonen ist es häufig nicht einfach, diese Frage zu beantworten. Die Meldepflicht richtet sich nach medizinischen Kriterien, die Juristen nicht beurteilen können, so Flavio Noto, Staatsanwalt des Kantons Basel-Stadt. Mit Unterstützung der Rechtsmedizin versucht die Staatsanwaltschaft zu klären, ob sorgfältig gehandelt wurde oder nicht. «War der Eingriff indiziert? Wurde über die möglichen Komplikationen aufgeklärt? Erfolgte die Behandlung lege artis? Wurde alles getan, um Komplikationen zu verhindern und um sich darauf einzustellen? Können alle Fragen mit Ja beantwortet werden, ist der Fall für mich erledigt", so Noto knapp. Ist dies nicht der Fall, leitet die Staatsanwaltschaft eine Untersuchung ein, um herauszufinden, inwiefern die Sorgfaltspflicht verletzt wurde. Dabei können die Behörden grundsätzlich auch Daten aus internen Fehlerberichterstattungssystemen, vielerorts bekannt als CIRS, beschlagnahmen: «Die sensiblen Einträge dieser Systeme werden jedoch kaum eingefordert, weil das gesellschaftliche Interesse an einem intakten Reportingsystem regelmässig höher ist als jenes an einer einzelnen Strafverfolgung", so Noto.

Weil in solchen Verfahren kollektive Verantwortung nicht existiert und jeder und jede aus dem Behandlungsteam als Einzelperson haftet, sollten Entscheide gerade auf Intensivstationen zur Absicherung niemals alleine getroffen werden, so die Rechtsmedizinerin Bettina Schrag aus Sion. Andreas Petrik, Rechtsanwalt aus St.Gallen, unterstreicht zudem die grosse Bedeutung der sofortigen Benachrichtigung der Vorgesetzten, damit die notwendigen Schritte schnell eingeleitet werden können.

\section{Wissen integrieren und Visiten optimieren}

Viel wichtiger ist es jedoch - das unterstreicht auch Tristan Uth aus dem Blickwinkel eines Komponisten und Dirigenten -, richtig miteinander zu kommunizieren, um Fehler möglichst zu verhindern. Dabei sollte es nicht unbedingt darum gehen, Wissen zu verbreiten, sagt Pierre Chopard, Leiter des Service Qualité des soins am HUG, weil dieses meistens bereits vorhanden ist. Viel wichtiger ist es, dafür zu sorgen, dass es auf der jeweiligen Abteilung auch wirklich integriert wird. Schliesslich bringt jegliches Wissen nichts, wenn es nicht angewandt wird.

Einen praktischen Versuch zur verbesserten Verständigung zwischen Ärzten, Pflegenden und auch Patienten starteten Peter Steiger und Daniela Lang-Eberle vom UniversitätsSpital Zürich im Jahr 2014. Nach dem Vorbild eines Lean Hospitals, in dem Veränderungen schnell und wirksam vor Ort umgesetzt werden, wurden alle Mitarbeiter der chirurgischen Intensivmedizin 
(CIM) - mit Ausnahme der leitenden Angestellten damit beauftragt, ein neues Visitenkonzept zu entwerfen, um Prozesse zu vereinfachen und die Kommunikation zu verbessern und zu beschleunigen. Ergänzt wurde die neue Visite durch ein sogenanntes HuddleBoard zur schnelleren und transparenteren Darstellung aktueller Patienteninformationen und Verantwortlichkeiten. Eine Evaluation unter den Angestellten im darauffolgenden Jahr fiel durchwegs positiv aus.

\section{Mit Benchmarking den eigenen Erfolg messen}

Ein Werkzeug zur Qualitätssicherung und -steigerung, das immer bedeutender wird, ist das Benchmarking. Hierbei werden unterschiedlichste Daten einer Intensivstation systematisch erfasst, analysiert und anschliessend verglichen, entweder mit den eigenen Vorjahresdaten oder mit denen einer anderen Intensivstation. Jukka Takala, Direktor und Chefarzt der Universitätsklinik für Intensivmedizin am Berner Inselspital, sieht einen Hauptgewinn des Benchmarkings darin, dass Intensivstationen dadurch Erkenntnisse über ihren Erfolg erhalten, die auf harten Fakten beruhen. So können sich Stationen gegenüber ihren Stakeholdern besser erklären. Der Patientenfluss lässt sich verbessern und Ressourcen können effizienter eingesetzt werden.

Das am weitesten verbreitete Benchmarking in der Schweizer Intensivmedizin geschieht mit dem Minimalen Datensatz der Intensivmedizin (MDSi) der SGI. So gehören die Schweiz und Finnland zu den wenigen Ländern, in denen Kennzahlen zum Behandlungsprozess in allen Intensivstationen erhoben und einer zentralen Datenbank zugeführt werden. Alle von der SGI zertifizierten Intensivstationen sind dazu verpflichtet, am MDSi teilzunehmen. Doch es gibt auch nicht zertifizierte Intensivstationen, die ihre Daten in den MDSi einspeisen. Laut Mark Kaufmann, dem Präsidenten der Kommission Datensatz der SGI, die für den MDSi zuständig ist, sind es pro Jahr etwa 90000 Datensätze, die so zusammenkommen. Ende Jahr erhalten alle teilnehmenden Stationen einen umfangreichen Bericht, in dem ihre Intensivstation dargestellt und mit Einheiten derselben Grösse verglichen wird. Diese Vergleichsmöglichkeit hat Regula Zürcher Zenklusen, der Chefärztin der Intensivstation am Hôpital neuchâtelois, etwa dazu verholfen, ein Personaldefizit an den Schweizer Durchschnitt anzugleichen.

\section{Vertrauen steigern und PDMS einführen}

In Zukunft soll der MDSi weiter verbessert werden, besonders mit Outcome-Daten, welche die Ergebnis- qualität einer Behandlung messen sollen. Jukka Takala warnt jedoch vor einem generellen Ausbau des Datensatzes, da die Qualität der erhobenen Kennzahlen mit der Grösse des Datensatzes zusammenhängt und ein grosser Datensatz tendenziell anfälliger ist für Fehler. Trotz des MDSi und Peer-Reviews, also Visitationen, welche im Zusammenhang mit der Zertifizierung von Intensivstationen durchgeführt werden, ist Benchmarking immer noch zu wenig etabliert. Das kann laut Nicolette de Keizer, Professorin für Medical Informatics an der Universität Amsterdam, durchaus daran liegen, dass hierzulande noch ein gewisses Vertrauen fehlt. Dieses ist wesentlich, damit unterschiedliche Stationen und Spitäler dazu bereit sind, ihre anonymisierten Datensätze miteinander zu vergleichen. Mit der steigenden Anzahl an teilnehmenden Intensivstationen würde sich das aber ändern, sagt De Keizer aus Erfahrung mit einem niederländischen Benchmarking-Projekt. Dies unterstreicht auch Philippe Eckert vom CHUV in Lausanne: "Man muss Vertrauen schaffen, und jemand muss im Benchmarking die Führung übernehmen.»

Eine weitere Grundvoraussetzung für erfolgreiches Benchmarking sind laut Björn Weiss von der Berliner Charité und Adrian Wäckerlin vom Kantonsspital Graubünden Patientendatenmanagementsysteme, bekannt als PDMS, die sämtliche Datensätze automatisch erfassen. So wird eine manuelle Dokumentation überflüssig, die Fehlerquote sinkt markant und die Stationen werden entlastet. In manchen Institutionen wurden solche PDMS vor weniger als zehn Jahren eingeführt, gewisse Stationen verfügen bis heute noch nicht über diese technische Infrastruktur, was zu einer Verzögerung in der Einführung von Benchmarkings führt.

Die Konstituierung der Qualitätskommission und die an der vergangenen Generalversammlung der SGI verabschiedete Qualitätscharta, in der Benchmarking eine bedeutende Rolle zukommt, sind wichtige Schritte zur Qualitätssicherung und -entwicklung auf Intensivstationen in der Schweiz. Grundlage bildet dabei die Sicherstellung einer hohen Patientensicherheit und eines effizienten Risikomanagements. Weil wir als Fachgesellschaft von der Bedeutung dieser Themen für die Intensivmedizin und das gesamte schweizerische Gesundheitswesen überzeugt sind, widmen wir das SGI Symposium 2018 inhaltlich der Patientensicherheit. Es wird am 27. April 2018 in Bern stattfinden.

Unter sgi-ssmi.ch finden Sie weitere Informationen zum MDSi, zur Qualitätskommission und zur Qualitätscharta. 